\title{
PARTIAL MOLECULAR CHARACTERIZATION OF COWPEA STUNT ISOLATES OF CUCUMBER MOSAIC VIRUS AND BLACK EYE COWPEA MOSAIC VIRUS FROM ARKANSAS AND GEORGIA (USA)
}

\author{
A. DIALLO'1.H., R.C. GERGERICH² and E.J. ANDERSON³ .
}

'University of Abobo-Adjame, UFR-SN, B.P. 801 Abidjan, COTE D'IVOIRE.

2University of Arkansas, Department of Plant Pathology, 217 PTSC Bldg, Fayetteville, ARKANSAS (USA) 72701.

${ }^{3}$ Pioneer Hi-Bred International, Inc., 6900 NW 62nd Ave., Johnston, IA 50131-0256.

\begin{abstract}
Partial molecular characterization of the coat protein of the cowpea stunt-causing isolates of Cucumber Mosaic Virus (CMV) from Arkansas and Georgia revealed that both isolates of CMV belong to CMV subgroup I and differ at eight nucleotides positions, resulting in two amino acids difference. There was only one amino acid difference for the Blackeye Cowpea Mosaic Virus (BICMV) isolates from both locations. Differences in the coat protein genes of CMV and BICMV isolates from Arkansas and Georgia could partially be responsible for the variation in the virus accumulation pattern. This is the first report on the taxonomic classification of the cowpea stunt disease-causing isolates of CMV and BICMV in Arkansas and Georgia based on nucleotides and amino acid sequences analysis.
\end{abstract}

Keywords : cowpea, Vigna unguiculata (L.) Walp. subsp. unguiculata, USA, Côte d'Ivoire.

\section{RESUME}

CARACTERISATION MOLECULAIRE PARTIELLE D'ISOLATS DE CMV ET BICMV ASSOCIES A LA MALADIE DU NANISME DU NIEBE DANS L'ARKANSAS ET LA GEORGIE (USA)

La caractérisation partielle des gènes de la protéine de capside des isolats de CMV associés à la maladie du nanisme du niébé provenant de l'Arkansas et de la Georgie a révélé que les deux isolats de CMV appartiennent au sous-group l et qu'ils diffèrent au niveau de huit nucléotides, ce qui se traduit par une différence de deux acides aminés. Pour BICMV, la différence entre les deux isolats porte seulement sur un acide aminé. Ces différences dans les gènes de la capside des isolats de CMV et de BICMV des deux différentes localités pourraient être partiellement responsables de la différence entre le mode d'accumulation de ces virus. Ceci constitue le premier travail sur la classification taxonomique, basé sur l'analyse des séquences d'acides nucléiques et d'acides aminés des isolats de CMV et de BICMV responsable du nanisme du niébé dans l'Arkansas et la Georgie.

Mots clés : Niébé, Vigna unguiculata (L.) Walp. sesp. unguiculata, USA, Côte d'Ivoire.

\section{INTRODUCTION}

Cowpea (Vigna unguiculata (L.) Walp. subsp. Unguiculata) is an important crop worldwide with a global production covering approximately 12.5 million ha of which, 8 million ha are in west and central Africa (Singh et al., 1997).

Cowpea stunt, a severe disease of cowpea, first reported in Georgia, Alabama and South Carolina (Pio-Ribeiro et al., 1978 ; Pio-Ribeiro and Kuhn,
1980), is caused by a synergistic interaction between blackeye cowpea mosaic virus (BICMV) and cucumber mosaic virus (CMV). Cowpea plants doubly infected with both viruses are severely stunted with small, blistered and malformed leaves.Stems and petioles of these infected plants become necrotic and show a significant reduction in the number of leaves and pods. Cowpea stunt caused important yield loss of $86.4 \%$ 
whereas only 2.5 and $14.2 \%$ reduction occurred with single infections with CMV and BICMV, respectively (Pio-Ribeiro et al., 1978). Cowpea stunt was more recently found in Magnolia County, Arkansas (Anderson et al., 1994).

Cucumber mosaic virus is the type virus of the cucumovirus group. It has a wide host range mostly composed of dicots. CMV can infect more than 800 plant species (Palukaitis et al., 1992). The virus is characterized by small, icosahedral particles of about $30 \mathrm{~nm}$ in diameter. It genome is composed of three genomic, positive-sense RNA molecules (RNAs 1, 2 and 3 ) and a subgenomic RNA (RNA 4) coding for the 24.5 Kilodalton $(\mathrm{Kd})$ coat protein (Matthews, 1991). The CMV coat protein (CP) is involved not only in symptom development, but also in the encapsidation of the RNAs as well as in aphid transmission (Mossop and Francki, 1977). Several strains of CMV have been isolated (Kapper and Waterworth, 1981; Rizos et al., 1992). In order to differentiate and classify these strains, several methods have been used: serology (Devergne and Cardin, 1983), nucleic acid hybridation (Gould and Symons, 1978; Piazzolla et al., 1979 ; Owen and Palukaitis, 1988) and peptide mapping of the coat protein gene (Edwards and Gonsalves, 1983). However, only nucleotide sequence analysis of the $\mathrm{CP}$ gene and the $3^{\prime}$ untranslated region was able to provide an accurate alternative differentiation between CMV strains and separate them into two subgroups, I and II (Quemada et al., 1989; Rizos et al., 1992).

Blackeye cowpea mosaic virus belongs to the most economically important group of plant viruses, the potyvirus group. These viruses particles are long and flexuous rod-shape, measuring approximately $12 \times 900 \mathrm{~nm}$. Potyviruses have their genome composed of a linear, single-stranded, positive-sense RNA, approximately 9.500 nucleotides (nt) in length. A genome-linked protein $(\mathrm{Vpg})$ is located at the $5^{\prime}$ terminus and at the $3^{\prime}$-end, there is a $200 \mathrm{nt}$ untranslated region (3'-UTR), followed by a polyA tail (Matthews, 1991). The virus genome consists of a large open reading frame. The coat protein gene is located at the 3 '-end. BICMV has been reported as the cowpea strain of bean yellow mosaic virus (BYMV) based on microagglutination test (Gay and Winstead, 1970 ; Harrison and Gudauskas, 1968a; Harrison and Gudauskas, 1968b ; Kuhn, 1964 ; Kuhn et al., 1965). However, based on comparative studies of cytological inclusions and host range, these viruses have been shown to be different (Edwardson et al., 1972 ; Zettler and Evans, 1972). BICMV and cowpea aphid-born mosaic virus (CaBMV), sometimes considered closely related or similar (Bock and Conti, 1974), have been differentiated based on their reactions on resistant cowpea cultivars (Taiwo et al., 1982). For a better differenciation and classification of potyviruses, methods based on coat protein and/ or 3'-UTR region sequences seem to give good results (Frenkel et al., 1989, 1991; Khan et al., 1990, 1993 ; Lana et al., 1988 ; Shukla and Ward, 1988 ; Shukla and Ward, 1989 ; Van der Vlugt et al., 1993).

Since cowpea stunt was first discovered in Georgia and later in Arkansas, it was important to compare the disease-causing isolates of CMV and BICMV from both geographical locations. In previous studies where the stunt-causing isolates of CMV and BICMV from Arkansas and Georgia were compared biologically, it was found that although all four types of mixed infections produced similar symptoms on cowpea plants, the viruses behaved differently based on their accumulation patterns in the leaves and stems (Diallo, 1998). The objective of this study was to molecularly characterize the Arkansas and Georgia cowpea stunt isolates of CMV and BICMV.

\section{MATERIALS AND METHODS}

\section{VIRUSES AND PLANTS}

The Arkansas cowpea isolates of BICMV $\left(B I C M V_{A R}\right)$ and $C M V\left(C M V_{A R}\right)$ were originally obtained from field samples taken in Columbia County in 1994. The Georgia isolates of cowpea stunt viruses $\left(B I C M V_{G A}\right.$ and $\left.C M V_{G A}\right)$ were provided by Dr. A. G. Gillaspie, Jr., USDA-ARS Genetic Resources Unit, University of Georgia, Griffin, GA. All viruses were maintained as dried infected tissues at $4{ }^{\circ} \mathrm{C}$ or in the cowpea cultivar 'Coronet' (Brantley, 1976) under greenhouse conditions with temperatures ranging from $20-$ $30{ }^{\circ} \mathrm{C}$. 'Coronet' cowpea plants were grown individually in 3-inch pots containing Redi-Earth $3 \mathrm{CP}$ potting mixture (Grace Sierra, Milpitas, CA).

\section{CHARACTERIZATION OF CMV AND BICMV COAT PROTEIN GENES}

Total nucleic acids from plants infected with $\mathrm{CMV}_{\mathrm{AR}}, \mathrm{CMV}_{\mathrm{GA}}, \mathrm{BICM}_{\mathrm{AR}}, \mathrm{BICMV}_{\mathrm{GA}}$ and healthy 
cowpea plants were extracted according to the procedure described by Pappu et al. (1993). Each plant sample was frozen in liquid nitrogen and ground in $300 \mu$ l of extraction buffer (2\% SDS, 0.1 M Tris, 0.002M EDTA, $\mathrm{pH}$ 8.0). The viral RNAs were extracted in phenol/ chloroformisoamyl alcohol (1:1). Crude RNA extracts were purified on a sephadex $\mathrm{G} 50$ column equilibrated with TE buffer (10 mM Tris, pH 7.5, 1 mM EDTA, $\mathrm{pH}$ 8.0). The nucleic acid eluants were collected and used for reverse transcriptase polymerase chain reaction (RT-PCR) amplification and cloning of the coat protein (CP) genes of all four isolates of CMV and BICMV according to the procedure described by Pappu et al. (1993). For CMV, two oligonucleotide primers were designed based on the published sequence of CMV strain $Q$ and FNY (Quemada et al., 1989). The entire CP gene including the 3 ' flanking sequence was amplified using the following upstream primer EA 39 (5'-TTC TCC GCG AGA TTG C-3') corresponding to positions 1167-1182 of the published sequence of FNY-CMV (c m $v$ FNY). The downstream primer, CMV3'CP (5'-CGT AAG CTG GAT GGA C3') corresponding to positions 2021-2036 of FNY-CMV was used. Using these two primers the predicted length of the CMV fragments is about 860 base pairs (bp).

Total nucleic acid extracts were heat denatured at $70{ }^{\circ} \mathrm{C}$ for $3 \mathrm{~min}$ and placed on ice. Each extract $(20 \mu \mathrm{l})$ was added to $73 \mu \mathrm{l}$ of the PCR mix [1X Taq DNA polymerase buffer (Promega, Madison, WI), 0.01M dithiothreitol (DTT), 0.04M dNTP mix, 2.5mM MgCl, 16 units of RNAsin (Promega), 20 units of AMV-reverse transcriptase (Promega), 2.5 unids of Taq DNA polymerase (Promega), and $100 \mathrm{~mol}$ of each oligonucleotide primer]. Samples were incubated at $41^{\circ} \mathrm{C}$ for $1 \mathrm{~h}$ for the synthesis of the first strand complementary DNA (cDNA) and placed in a thermal cycler (Barnstead Thermolyne). Initial denaturation was done at $96^{\circ} \mathrm{C}$ for 2 min. Thirtyfive cycles were performed with periods of $1 \mathrm{~min}$ for annealing at $44^{\circ} \mathrm{C}, 1 \mathrm{~min}$ for synthesis at 72 ${ }^{\circ} \mathrm{C}$ and $1 \mathrm{~min}$ for melting at $94.5^{\circ} \mathrm{C}$. The reverse transcriptase (RT-PCR) products were analysed by a $1 \%$ agarose gel electro-phoresis and visualized by ethydium bromide staining.

The entire CP gene and the 3'-untranslated regions (3'-UTR) of BICMV isolates from Arkansas and Georgia were amplified using a degenerate primer EA 13 (5'-AAG ATT GAA GAG TTA GC-3') designed based on the published sequence of BICMV strain W (Khan et al., 1993) corresponding to positions (1-17) on the viral polymerase gene portion of the published sequence and an oligo-dT primer. The predicted length of the RT-PCR fragment was approximately $1200 \mathrm{bp}$. Total nucleic acids were denatured at $70^{\circ} \mathrm{C}$ for $3 \mathrm{~min}$, and $55.3 \mu$ added to tubes containing $\beta 6.9 \mu$ l of PCR mix (same as before) and $100 \mathrm{~mol}$ of each primer. The reactions were incubated at $42^{\circ} \mathrm{C}$ for $1 \mathrm{~h}$ for the synthesis of the first strand cDNA. Thirty cycles were run in a thermal cycler with periods of $45 \mathrm{~s}$ at $93.5^{\circ} \mathrm{C}$ for denaturation, $45 \mathrm{~s}$ at $36{ }^{\circ} \mathrm{C}$ for annealing and $1 \mathrm{~min}$ at $72^{\circ} \mathrm{C}$ for extension. The RT-PCR fragments were analysed by a $1 \%$ agarose gel electrophoresis.

\section{CLONINGAND SEQENCING OF CMV AND BLCMV COAT PROTEIN GENES}

The RT-PCR fragments of CMV and BICMV isolates from Arkansas and Georgia were blunt-ended with DNA polymerase I (Klenow fragment), extracted from $0.8 \%$ low melting point agarose gel in $0.05 \mathrm{M}$ Tris-borate/EDTA electrophoresis buffer (TBE), and phosphorylated. CMV and BICMV fragments were ligated into the Sma I site of pUC118 and pUC119 plasmids respectively and transformed into competent Escherichia coli DH5a cells. Transformed bacteria were cultured on solid 2XYT medium containing $75 \mu \mathrm{g} / \mathrm{ml}$ ampicillin, $40 \mu \mathrm{g} / \mathrm{ml} \mathrm{X-gal} \mathrm{(5-bromo-4-chloro-3-}$ indolyl-b-D-galactoside, United States Biochemical) and $8 \mu \mathrm{g} / \mathrm{ml}$ IPTG (isopropylthiob-D-galactoside, United States Biochemical) and incubated at $37^{\circ} \mathrm{C}$ for $16 \mathrm{~h}$. Recombinants were screened by blue and white colour reaction. White colonies were cultured in 2XYT liquid medium containing ampicillin. Plasmids were digested with restricted enzymes EcoRI and Pstl according to the supplier's recommendations. Inserts were detected by agarose gel electrophoresis. Plasmids with inserts from selected clones were sequenced by the dideoxynucleotide chain termination method (Sanger et al., 1977) using the Sequenase v.2.0 kit (USB, Cleveland, OH) and the dideoxynucleotide sequencing method. At least 2 independent clones from each virus were sequenced in both directions. Sequence data were analysed and compared using PC/Gene v.6.85 (Intelligenetics, Campbell, CA). 


\section{RESULTS}

\section{PARTIAL MOLECULAR CHARACTERIZATION OF THE ARKANSAS AND GEORGIA ISOLATES OF CMV}

PCR-amplified viral products of $\mathrm{CMV}_{\mathrm{AR}}$ and $\mathrm{CMV}_{\mathrm{GA}}$ were analysed by agarose gel electrophoresis. Both viral coat protein genes displayed identical migration patterns and sizes which corresponded to the expected size of 860 nucleotides (Diallo,1998). The nucleotide sequence comparison of the coat protein genes and portion of the 3' flanking regions of the $C M V_{A R}$ and $C M V_{G A}$ isolates showed that $\mathrm{CMV}_{\mathrm{AR}}$ differed from $\mathrm{CMV}_{\mathrm{GA}}$ isolate at only eight positions (figure 1), with seven of the differences located in the coding region. There were 14 nucleotide differences b. tw.en c m $v$ AR and CMVFNY and 12 between $\mathrm{CMV}_{\mathrm{GA}}$ and CMVFNy. The CP fragment nucleotide sequences homology of the two CMV isolates was $99 \%, 90 \%$ with subgroup I CMV and only 77-80 \% with subgroup II CMV. The CMV CP genes encoded the predicted 218 amino acid residues for $\mathrm{CMV}_{\mathrm{AR}}, \mathrm{CMV}_{\mathrm{GA}}$ and $\mathrm{CMV}_{\mathrm{FNY}}$. The deduced amino acid sequences of $C M V_{A R}$ and $\mathrm{CMV}_{\mathrm{GA}}$ revealed only two amino acid differences located at positions 61 and 124 (figure 2). $C M V_{F N Y}$ on the other hand differed from $\mathrm{CMV}_{\mathrm{AR}}$ and $\mathrm{CMV}_{\mathrm{GA}}$ at 14 and 12 amino acid residues, respectively, with the differences not restricted to any specific area of the coat protein gene.

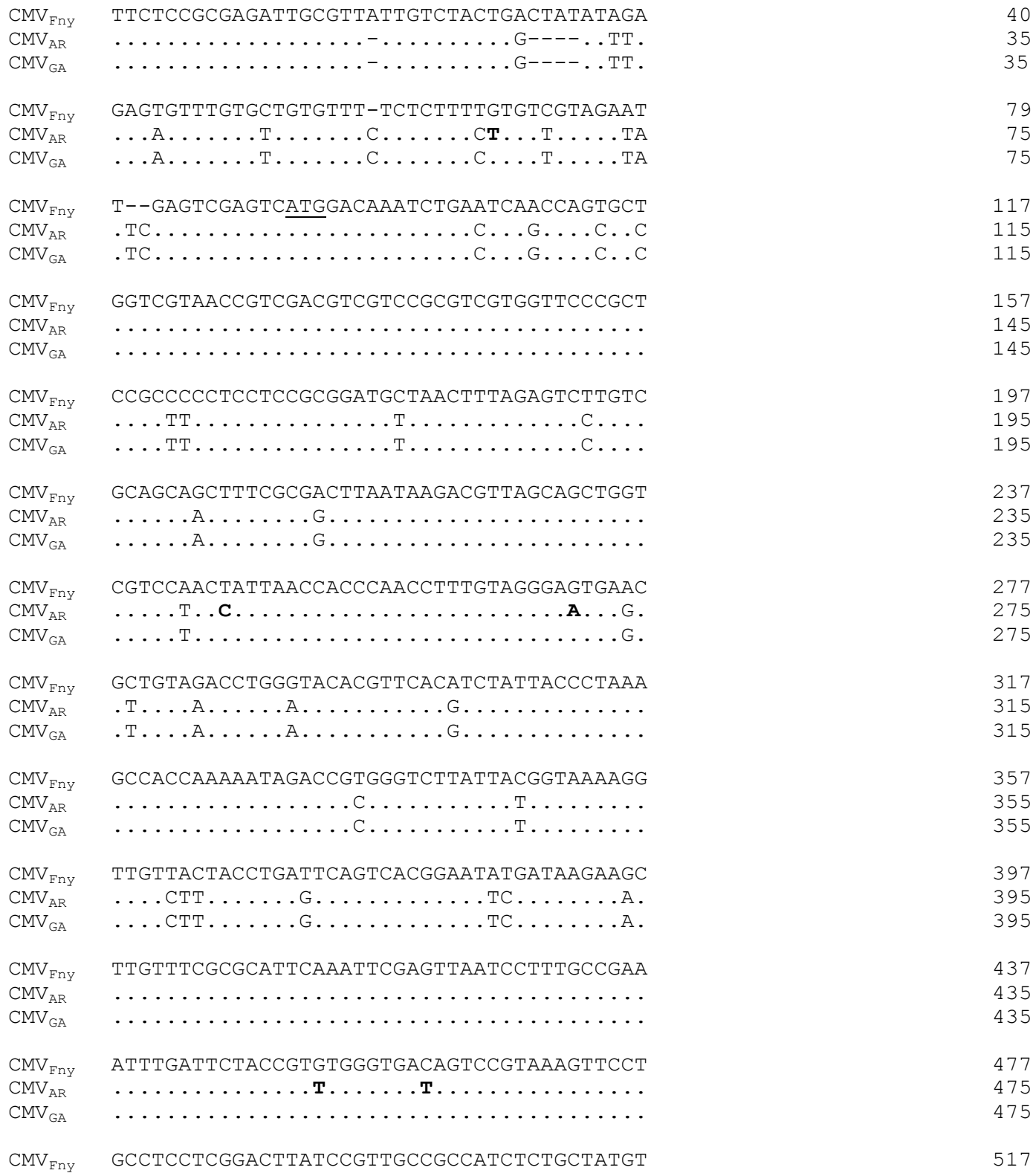




\begin{tabular}{|c|c|c|}
\hline $\begin{array}{l}\mathrm{CMV}_{\mathrm{AR}} \\
\mathrm{CMV}_{\mathrm{GA}}\end{array}$ & 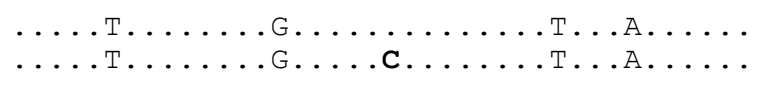 & $\begin{array}{l}515 \\
515\end{array}$ \\
\hline $\mathrm{CMV}_{\text {Fny }}$ & TCGCGGACGGAGCCTCACCGGTACTGGTTTATCAGTATGC & 557 \\
\hline $\mathrm{CMV}_{\mathrm{AR}}$ & 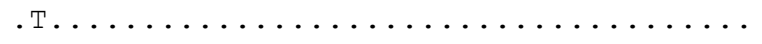 & 555 \\
\hline $\mathrm{CMV}_{\mathrm{GA}}$ & 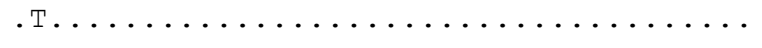 & 555 \\
\hline $\mathrm{CMV}_{\text {Fny }}$ & CGCATCTGGAGTCCAAGCCAACAACAAACTGTTGTATGAT & 597 \\
\hline $\mathrm{CMV}_{\mathrm{AR}}$ & 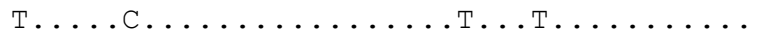 & 595 \\
\hline $\mathrm{CMV}_{\mathrm{GA}}$ & 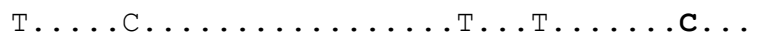 & 595 \\
\hline $\mathrm{CMV}_{\text {Fny }}$ & СтTTCGGCGATGCGCGCTGATATAGGTGACATGAGAAAGT & 637 \\
\hline $\mathrm{CMV}_{\mathrm{AR}}$ & $\ldots \ldots$. . . . . . . . . . . . & 635 \\
\hline $\mathrm{CMV}_{\mathrm{GA}}$ & $\ldots$. АС $\ldots$. . . . Т . . . . С. . . & 635 \\
\hline $\mathrm{CMV}_{\text {Fny }}$ & ACGCCGTCCTCGTGTATTCAAAAGACGATGCGCTCGAGAC & 677 \\
\hline $\mathrm{CMV}_{\mathrm{AR}}$ & $\ldots \ldots$.............A.A. Т.. Т. & 675 \\
\hline $\mathrm{CMV}_{\mathrm{GA}}$ & $\ldots \ldots$. . . . . . . . A.A. . . & 675 \\
\hline $\mathrm{CMV}_{\text {Eny }}$ & GGACGAGCTAGTACTTCATGTTGACATCGAGCACCAACGC & 717 \\
\hline $\mathrm{CMV}_{\mathrm{AR}}$ &  & 5 \\
\hline $\mathrm{CMV}_{\mathrm{GA}}$ & $\ldots \mathrm{T} \ldots \ldots \mathrm{G} \ldots \ldots \mathrm{C} \ldots \mathrm{C} \ldots \mathrm{T} \ldots \mathrm{C}$ & 715 \\
\hline $\mathrm{CMV}_{\text {Fny }}$ & АTTCCCACATCTGGAGTGCTCCCAGTCTGATTCCGTGTT- & \\
\hline $\mathrm{CMV}_{\mathrm{AR}}$ & $\ldots \ldots \ldots \ldots \ldots \ldots \ldots \ldots \ldots \ldots$ & 75 \\
\hline $\mathrm{CMV}_{\mathrm{GA}}$ & 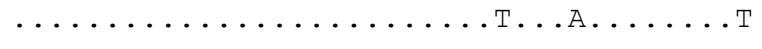 & 75 \\
\hline $\mathrm{CMV}_{\text {Fny }}$ & СССАGААTССТСССТCCGATCTCTGTGGCGGGACGTGAGT & 79 \\
\hline $\mathrm{CMV}_{\mathrm{AR}}$ & $\ldots$..........AT.T............ & 79 \\
\hline $\mathrm{CMV}_{\mathrm{GA}}$ & 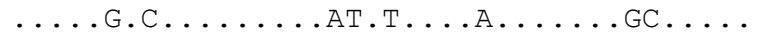 & \\
\hline $\mathrm{CMV}_{\text {Fny }}$ & TGGCAGTTCTGCTATAAACTGTCTGAAGTCACTAAA-CGT & 83 \\
\hline $\mathrm{CMV}_{\mathrm{AR}}$ & $\ldots \ldots$.........................$^{--}$ & 83 \\
\hline $\mathrm{CMV}_{\mathrm{GA}}$ & $\ldots \ldots$...АТС .................- & 8 \\
\hline $\mathrm{CMV}_{\text {Eny }}$ & TTTTTACGGTGAACGGGTTGTCCATCCAGCTTACG & 8 \\
\hline $\mathrm{CMV}_{\mathrm{AR}}$ & $--\ldots G \ldots \ldots \ldots \ldots \ldots \ldots \ldots$ & \\
\hline $\mathrm{CMV}_{\mathrm{GA}}$ & $--\ldots G \ldots \ldots \ldots \ldots \ldots \ldots \ldots \ldots$ & 0 \\
\hline
\end{tabular}

Figure 1 : Comparison of the nucleotide sequences of the coat protein and untranslated regions of the published data for CMVFNY (Quemada et al., 1989), the Arkansas isolate of CMV (CMVAR) and the Georgia isolate of CMV (CMVGA).

Dots (...) indicate nucleotides identical to CMVFNY sequences and dashes (---) represent missing residues. The ATG and AGT codons corres pond to the initiation and termination sites for the putative CMV coat protein open reading frame. Nucleotide differences between CMVAR and CMVGA are in bold.

Comparaison des séquences de nucléotides des régions de la protéine de capside et noncodante du CMVFNY publié (Quemada et al., 1989), de l'isolat de CMV de l'Arkansas (CMVAR) et de l'isolat de CMV de la Georgie (CMVGA).

Les ( ...) indiquent les nucléotides identiques aux séquences de CMVFNY et les tirets (---) représentent les résidus manquants. Les codons ATG et AGT correspondent aux sites d'initiation et de terminaison du cadre de lecture. Les différen ces en nucléotides entre CMVAR et CMVGA sont en gras. 


\begin{tabular}{|c|c|c|}
\hline $\mathrm{CMV}_{\mathrm{Fny}}$ & MDKSESTSAGRNRRRRPRRGSRSAPSSADANFRVLSQQLS & 40 \\
\hline $\mathrm{CMV}_{\mathrm{AR}}$ & $\ldots$. D.A...................... & 40 \\
\hline $\mathrm{CMV}_{\mathrm{GA}}$ & 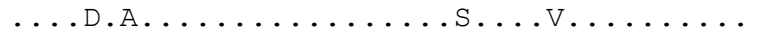 & 40 \\
\hline $\mathrm{CMV}_{\text {Fny }}$ & RLNKTLAAGRPTINHPTFVGSERCRPGYTFTSITLKPPKI & 80 \\
\hline $\mathrm{CMV}_{\mathrm{AR}}$ & 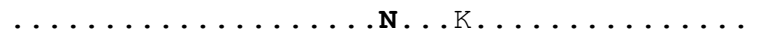 & 80 \\
\hline $\mathrm{CMV}_{\mathrm{GA}}$ & 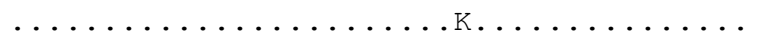 & 0 \\
\hline $\mathrm{CMV}_{\text {Fny }}$ & DRGSYYGKRLLLPDSVTEYDKKLVSRIQIRVNPLPKFDST & 120 \\
\hline $\mathrm{CMV}_{\mathrm{AR}}$ & 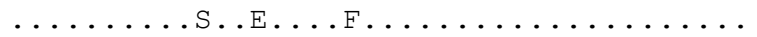 & \\
\hline $\mathrm{CMV}_{\mathrm{GA}}$ & $\ldots \ldots \ldots$. & 1 \\
\hline $\mathrm{CMV}_{\mathrm{FnY}}$ & VWVTVRKVPASSDLSVAAISAMFADGAS PVLVYQYAASGV & 0 \\
\hline $\mathrm{CMV}_{\mathrm{AR}}$ & 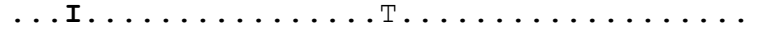 & 0 \\
\hline $\mathrm{CMV}_{\mathrm{GA}}$ & 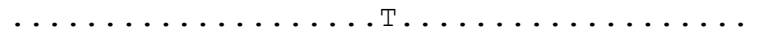 & \\
\hline $\mathrm{CMV}_{\text {Fny }}$ & QANNKLLYDLSAMRADIGDMRKYAVLVYSKDDALETDELV & 0 \\
\hline $\mathrm{CMV}_{\mathrm{AR}}$ & $\ldots \ldots \ldots$. $\ldots \ldots$. & 0 \\
\hline $\mathrm{CMV}_{\mathrm{GA}}$ & $\ldots \ldots \ldots$. $\ldots \ldots \ldots \ldots$. & \\
\hline $\mathrm{CMV}_{\text {Fny }}$ & LHVDIEHQRIPTSGVLPV & \\
\hline & $\ldots \ldots \ldots \ldots$ & \\
\hline $\mathrm{V}_{\mathrm{GA}}$ & $\ldots \ldots \ldots \ldots$ & \\
\hline
\end{tabular}

Figure 2 : Comparison of the deduced amino acid sequences of the coat protein genes of the published data for CMVFNY (Quemada et al., 1989), the Arkansas isolate of CMV (CMVAR), and the Georgia isolate of CMV (CMVGA).

Dots (...) indicate amino acids identical to the CMVFNY sequence. Amino acid differences between CMVAR and CMVGA are in bold.

Comparaison des séquences d'acides aminés des gènes de la protéine de capside de CMVFNY publié (Quemada et al., 1989), de l'isolat de CMV de l'Arkansas (CMVAR), et de l'isolat de CMV de la Georgie (CMVGA).

Les points (...) indiquent les acides aminés identiques à la séquences de CMVFNY. Les différences d'acides aminés entre CMVAR et CMVGA sont en gras.

\section{PARTIAL MOLECULAR CHARACTERIZATION OF THEAR- KANSAS AND GEORGIA ISOLATES OF BLCMV}

PCR-amplified products of BICMV $V_{A R}$ and $B V_{G A}$ were analysed by agarose gel electrophoresis. Similar migration patterns and sizes (approximately 1200 bases) corresponding to the expected sizes were observed for both BICMV isolates (Diallo, 1998). Nucleotide sequence comparisons between the Arkansas and Georgia isolates of BICMV showed only one nucleotide difference located at position 281 (99 $\%$ homology). Alignment with BICMVw (Khan et al., 1993) indicated only one nucleotide difference with $B I C M V_{G A}$ at that position (figure 3). The amino acid sequences of both isolates of BICMV (from Arkansas and Georgia) were deduced from the nucleotide sequences.
The predicted amino acid sequence data for the coat protein genes of $B I C M V_{A R}, B I C M V_{G A}$ and $B I C M V_{w}$ were aligned (figure 4). Both predicted $B I C M V_{A R}$ and $B I C M V_{G A}$ coat proteins were identical in size (239 amino acid residues) while the deduced amino acid sequence of BICMVw was a few amino acids longer (244 residues). The Arkansas and Georgia isolates of BICMV differed by only one amino acid located at position 19 where the glutamine on $\mathrm{BICMV}_{\mathrm{AR}}$ is replaced by a proline on $\mathrm{BICMV}_{\mathrm{GA}}$. At that same position $B I C M V_{w}$ had also a $Q$ residue similar

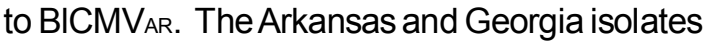
differed from BICMVw at 10 and 11 amino acid positions, respectively. The 3 ' end of all three isolates of BICMV displayed some important differences. There seemed to be a five amino acids deletion or insertion depending on the virus isolate (figure 4). The overall similarity of the amino acid sequence of $B I C M V_{A R}, B I C M V_{G A}$ and BICMVw was $93 \%$. 


\begin{tabular}{|c|c|c|}
\hline $\mathrm{BlCMV}_{\mathrm{W}}$ & AAGATTGAAGAGTTAGCCAAGTATCTTGAAGTGTTTGACT & 40 \\
\hline $\mathrm{BlCMV} \mathrm{AR}_{\mathrm{AR}}$ & $\ldots \ldots \ldots \ldots$ A A . A. C. G.... C.C.T. & 40 \\
\hline $\mathrm{B} \perp \mathrm{CMV}_{\mathrm{GA}}$ &  & 40 \\
\hline $\mathrm{BlCMV}_{\mathrm{W}}$ & TTGACTATGATGTAGGATGCGGAGAATCTGTGCACCTACA & 80 \\
\hline $\mathrm{B} I \mathrm{CMV}_{\mathrm{AR}}$ & 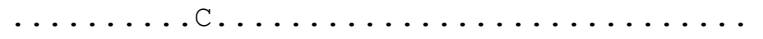 & 80 \\
\hline $\mathrm{BlCMV} V_{\mathrm{GA}}$ & 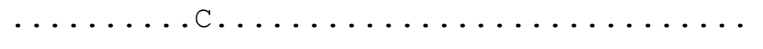 & 80 \\
\hline $\mathrm{BlCMV}_{\mathrm{W}}$ & ATCTGGAACTGGACAGCCGCAACCACCAATAGTGGATGCT & 120 \\
\hline $\mathrm{B} l \mathrm{CMV}_{\mathrm{AR}}$ & $\ldots$ A........................ & 120 \\
\hline $\mathrm{B} I \mathrm{CMV} V_{\mathrm{GA}}$ & $\ldots$. . . . . . . . . . . . . . . & 120 \\
\hline $\mathrm{BlCMV}_{\mathrm{W}}$ & GGTGTGGATGCTGGAAAGGACAAGAGAGAGAGAAGCAATA & 160 \\
\hline $\mathrm{B} l \mathrm{CMV}_{\mathrm{AR}}$ & 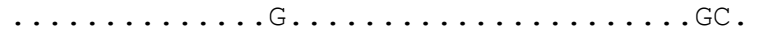 & 160 \\
\hline $\mathrm{B} l \mathrm{CMV}_{\mathrm{GA}}$ & $\ldots \ldots \ldots \ldots \ldots \ldots \ldots \ldots$ GC & 160 \\
\hline $\mathrm{BlCMV}_{W}$ & GAGGAAAAGACCCTGAAAGCAGGGAGGGGTCAGTAAACAA & 200 \\
\hline $\mathrm{B} l \mathrm{CMV}_{\mathrm{AR}}$ & $\ldots \ldots \ldots \ldots \ldots$. . . . . . . GG... & 200 \\
\hline $\mathrm{BlCMV} \mathrm{GA}_{\mathrm{G}}$ & $\ldots \ldots \ldots \ldots \ldots$. A $\ldots$. T...GG . . & 200 \\
\hline $\mathrm{BlCMV}_{\mathrm{W}}$ & CAACCGTGGTGCAGGGGATTCAACAATGAGAGACAAGGAT & 240 \\
\hline $\mathrm{B} \perp \mathrm{CMV}_{\mathrm{AR}}$ & $\ldots \ldots \ldots \ldots$. . . . . . . . . & 240 \\
\hline $\mathrm{BlCMV} V_{\mathrm{GA}}$ & $\cdots \cdots \cdots \cdots$ A $\cdots \cdots \cdots \cdots \cdots$ & 240 \\
\hline $\mathrm{BlCMV}_{W}$ & GTGAACGCAGGCTCCAGGGGAAAAGTTGTCCCGCGGCTTC & 280 \\
\hline $\mathrm{B} I \mathrm{CMV}_{\mathrm{AR}}$ & 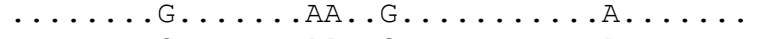 & 280 \\
\hline$B l C M V_{G A}$ &  & 280 \\
\hline $\mathrm{BlCMV}$ W & AAAAGATCACAAAAAGGATGAACTTGCCCATGGTGAAAGG & 320 \\
\hline $\mathrm{B} I \mathrm{CMV}_{\mathrm{AR}}$ & 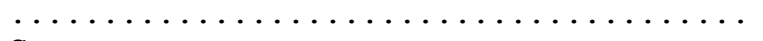 & 320 \\
\hline $\mathrm{B} l \mathrm{CMV} \mathrm{GA}_{\mathrm{A}}$ & 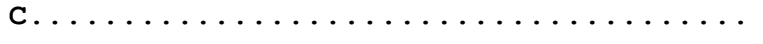 & 320 \\
\hline $\mathrm{BlCMV}_{\mathrm{W}}$ & GAATGTTATTTTAAATCTAGATCATCTGTTGGATTACAAG & 360 \\
\hline $\mathrm{BlCMV} \mathrm{CR}_{\mathrm{AR}}$ & $\ldots \ldots$. . . . . . А . . . & 360 \\
\hline$B l C M V_{G A}$ & 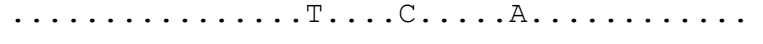 & 360 \\
\hline $\mathrm{BlCMV} \mathrm{W}_{\mathrm{W}}$ & CCAGTTCAAАСTGACСTTTTTAACACAAGAGCAACAAGGG & 400 \\
\hline $\mathrm{B} l \mathrm{CMV}_{\mathrm{AR}}$ & $\ldots$. AA..... T... . . . . . . A & 400 \\
\hline $\mathrm{BlCMV} \mathrm{GA}_{\mathrm{G}}$ & $\ldots$.АА $\ldots$. Т. . С . . . . . A.A & 400 \\
\hline $\mathrm{BlCMV}_{\mathrm{W}}$ & ACCAGTTTGAAATGTGGTACAATGCTGTGAAGGGCGAGTA & 440 \\
\hline $\mathrm{B} l \mathrm{CMV}_{\mathrm{AR}}$ & 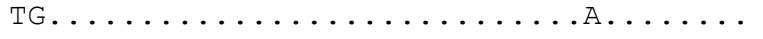 & 440 \\
\hline $\mathrm{B} \perp \mathrm{CMV}_{\mathrm{GA}}$ & 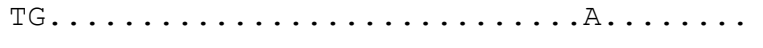 & 440 \\
\hline $\mathrm{BlCMV}$ W & TGAAATAGATGATGATCAGATGTCAATTGTAATGAACGGA & 480 \\
\hline $\mathrm{B} l \mathrm{CMV}_{\mathrm{AR}}$ & $\ldots$ G..................... & 480 \\
\hline $\mathrm{B} l \mathrm{CMV} V_{\mathrm{GA}}$ & $\ldots$ G.......CA. A........... . & 480 \\
\hline $\mathrm{BlCMV}_{\mathrm{W}}$ & TTCATGGTGTGGTGTATTGACAATGGCACTTCACCGGATG & 520 \\
\hline $\mathrm{BlCMV} \mathrm{AR}_{\mathrm{AR}}$ & 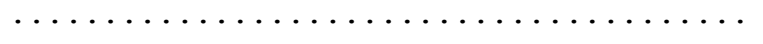 & 520 \\
\hline $\mathrm{B} l \mathrm{CMV}_{\mathrm{GA}}$ & $\cdots \cdots \cdots \cdots \cdots \cdots \cdots \cdots \cdots \cdots \cdots \cdots \cdots \cdots \cdots$ & 520 \\
\hline $\mathrm{BlCMV}_{\mathrm{W}}$ & TGAATGGTACATGGGTGATGATGGATGGAGATGAGCAAGT & 560 \\
\hline $\mathrm{B} I \mathrm{CMV}_{\mathrm{AR}}$ & 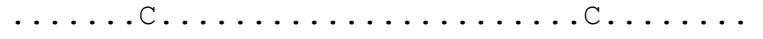 & 560 \\
\hline$B l C M V_{G A}$ & $\ldots \ldots$. $\ldots \ldots \ldots \ldots \ldots \ldots \ldots$ & 560 \\
\hline $\mathrm{BlCMV}_{\mathrm{W}}$ & TGAATACCCACTCAAACCAATGGTTGAAAATGCAAAGCCA & 600 \\
\hline $\mathrm{B} \perp \mathrm{CMV}_{\mathrm{AR}}$ & 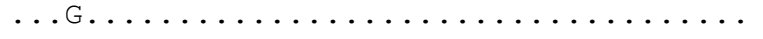 & 600 \\
\hline $\mathrm{B} \perp \mathrm{CMV}_{\mathrm{GA}}$ & 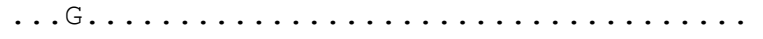 & 600 \\
\hline $\mathrm{BlCMV}_{W}$ & АСАСТCCGTCAАATCATGCACCATTTCTCAGATGCAGCTG & 640 \\
\hline $\mathrm{B} l \mathrm{CMV}_{\mathrm{AR}}$ & $\cdots \cdots \cdots \cdots \cdots \cdots \cdots \cdots \cdots$ & 640 \\
\hline $\mathrm{BlCMV}_{\mathrm{GA}}$ & $\cdots \cdots \cdots \cdots \cdots \cdots \cdots \cdots \cdots$ & 640 \\
\hline $\mathrm{BlCMV}_{\mathrm{W}}$ & AAGCATACATTGAGATGAGAAATTCCGAAAGGCCGTACAT & 680 \\
\hline $\mathrm{B} I \mathrm{CMV}_{\mathrm{AR}}$ & $\ldots \ldots \ldots \ldots \ldots \ldots \ldots \ldots \ldots \ldots \ldots \ldots \ldots \ldots$ & 680 \\
\hline $\mathrm{B} l \mathrm{CMV}_{\mathrm{GA}}$ & $\ldots \ldots \ldots \ldots \ldots \ldots \ldots \ldots \ldots \ldots \ldots \ldots \ldots \ldots$ & 680 \\
\hline
\end{tabular}




\begin{tabular}{|c|c|c|}
\hline $\mathrm{BlCMV}_{\mathrm{W}}$ & GCCTAGGTACGGACTACTTCGGAATTTGAGGGATAAAAAT & 720 \\
\hline $\mathrm{BlCMV}_{\mathrm{AR}}$ & 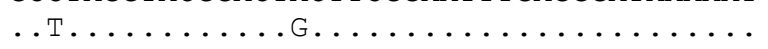 & 720 \\
\hline $\mathrm{BlCMV}_{\mathrm{GA}}$ & 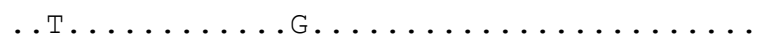 & 720 \\
\hline $\mathrm{BlCMV}_{\mathrm{W}}$ & СTAGCTCGCTACGCTTTTGATTTCTATGAGGTGACATCAA & 760 \\
\hline $\mathrm{BlCMV} \mathrm{V}_{\mathrm{AR}}$ & 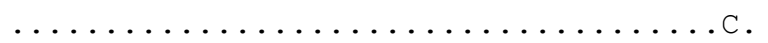 & 760 \\
\hline $\mathrm{BlCMV}_{\mathrm{GA}}$ & 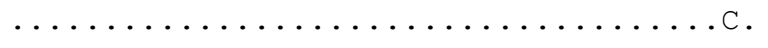 & 760 \\
\hline $\mathrm{BlCMV}_{\mathrm{W}}$ & AAACATCGGATCGAGCCAGAGAAGCAGTAGCACAGATGAA & 800 \\
\hline$B I \mathrm{CMV}_{\mathrm{AR}}$ & 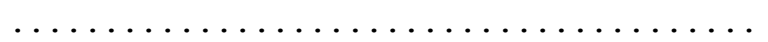 & 800 \\
\hline $\mathrm{BlCMV}_{\mathrm{GA}}$ & 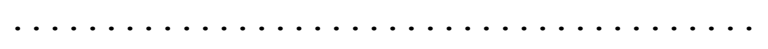 & 800 \\
\hline $\mathrm{BlCMV}_{\mathrm{W}}$ & GGCAGCAGCCCTCAGCAACGTTAGCAGCAAGTTGTTTGGA & 840 \\
\hline $\mathrm{BlCMV} \mathrm{V}_{\mathrm{AR}}$ & 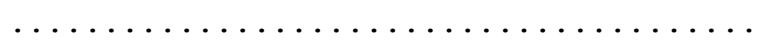 & 840 \\
\hline $\mathrm{BlCMV}_{\mathrm{GA}}$ & 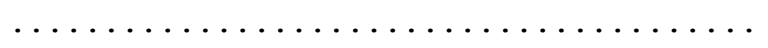 & 840 \\
\hline $\mathrm{BlCMV}_{\mathrm{W}}$ & CTTGACGGTAATGTTGCAACAACCAGCGAGAATACTGAAA & 880 \\
\hline $\mathrm{BlCMV} \mathrm{V}_{\mathrm{AR}}$ & $\ldots \ldots$. $\ldots \ldots \ldots \ldots \ldots \ldots \ldots \ldots \ldots \ldots$ & 880 \\
\hline $\mathrm{BlCMV}_{\mathrm{GA}}$ & $\ldots \ldots$ т................. & 880 \\
\hline $\mathrm{BlCMV}_{\mathrm{W}}$ & GGCACACTGCAAGGGACGTTAACCAAAACATGCACACACT & 920 \\
\hline $\mathrm{BlCMV} \mathrm{V}_{\mathrm{AR}}$ & 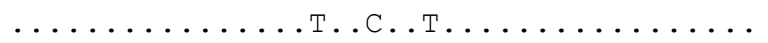 & 920 \\
\hline $\mathrm{BlCMV}_{\mathrm{GA}}$ & $\ldots \ldots \ldots \ldots \ldots$ т.скт......... & 920 \\
\hline $\mathrm{BlCMV}_{\mathrm{W}}$ & TCTTGGCATGGTTCTCC-GCAGTAAAGGTTGGGTAAACTG & 960 \\
\hline $\mathrm{BlCMV} \mathrm{V}_{\mathrm{AR}}$ & $\ldots \ldots \ldots$ GC.C............ & 960 \\
\hline $\mathrm{BlCMV}_{\mathrm{GA}}$ & $\ldots \ldots \ldots$ GC.C.............. & 960 \\
\hline $\mathrm{BlCMV}_{\mathrm{W}}$ & ACCACAGTTAGCATCTCGCGTCGCTGAATAATTTCATATA & 1000 \\
\hline $\mathrm{BlCMV} \mathrm{V}_{\mathrm{AR}}$ & $\ldots \ldots \ldots \ldots \ldots$ та. & 1000 \\
\hline $\mathrm{BlCMV} \mathrm{GA}_{\mathrm{A}}$ & 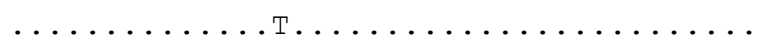 & 1000 \\
\hline $\mathrm{BlCMV}_{\mathrm{W}}$ & GTAATCTTTTATGTTCTCTTTAGTTTCTGTGTGGTTTTAC & 1040 \\
\hline $\mathrm{BlCMV} \mathrm{V}_{\mathrm{AR}}$ & $\ldots \ldots \ldots \ldots \ldots$ т. $\ldots \ldots \ldots$ А. . . & 1040 \\
\hline $\mathrm{BlCMV}_{\mathrm{GA}}$ & $\ldots \ldots \ldots \ldots \ldots$ т. $\ldots \ldots \ldots$ А. . . АС & 1040 \\
\hline $\mathrm{BlCMV}_{\mathrm{W}}$ & CACCTTTGTGTTACTATTGTGATAGCGTGGTTAGTCCACC & 1080 \\
\hline$B l \mathrm{CMV}_{\mathrm{AR}}$ & 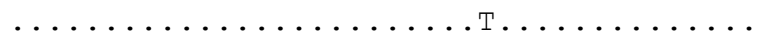 & 1080 \\
\hline $\mathrm{BlCMV}_{\mathrm{GA}}$ & 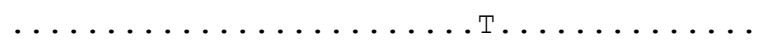 & 1080 \\
\hline $\mathrm{BlCMV}_{\mathrm{W}}$ & AACATATTGTGAGTACTTTATGTTTATGAGTAAGCCGGAA & 1120 \\
\hline $\mathrm{BlCMV} \mathrm{V}_{\mathrm{AR}}$ & $\ldots \ldots \ldots \ldots \ldots \ldots \ldots \ldots \ldots \ldots \ldots \ldots \ldots$ & 1120 \\
\hline $\mathrm{BlCMV}_{\mathrm{GA}}$ & $\ldots \ldots \ldots \ldots \ldots \ldots \ldots \ldots \ldots \ldots \ldots \ldots \ldots$ & 1120 \\
\hline $\mathrm{BlCMV}_{\mathrm{W}}$ & GAACCATTGCAATGGTGAGGGCATGCAGAGTGATTTGATC & 1160 \\
\hline $\mathrm{BlCMV} \mathrm{V}_{\mathrm{AR}}$ &  & 1160 \\
\hline $\mathrm{BlCMV}_{\mathrm{GA}}$ & 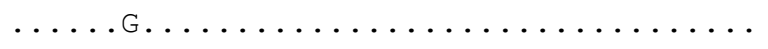 & 1160 \\
\hline $\mathrm{BlCMV}_{\mathrm{W}}$ & ATGTGTCATGAAGTAGCTACGGCAATGTTTGTTGTT-- & 1198 \\
\hline $\mathrm{BICMV} \mathrm{V}_{\mathrm{AR}}$ & $. C . C . C \ldots \ldots$. ...C..........CC & 1198 \\
\hline $\mathrm{BlCMV}_{\mathrm{GA}}$ &. C.C.C....G..............CC & 1198 \\
\hline
\end{tabular}

Figure 3 : Comparison of the nucleotide sequences of the coat protein genes, 3' untranslated regions and portion of the polymerase of the published data for BICMVW (Khan et al., 1993), the Arkansas solate of BICMV (BICMVAR), and the Georgia isolate of BICMV (BICMVGA).

Dots (...) indicate nucleotide identical to the BICMVW sequence, and dashes (---) represent missing residues. The ATG and TAG codons correspond to the initiation and termination sites for the putative BICMV coat protein open reading frame. Nucleotide differences between the Arkansas and Georgia isolates of BICMV are in bold.

Comparaison des séquences de nucléotides des gènes de la protéine de capside et d'une partie de la polymérase du BICMVW publié (Khan et al., 1993), de l'isolat de BICMV de l'Arkansas (BICMVAR) et de l'isolat de BICMV de la Georgie (BICMVGA).

Les (...) indiquent les nucléotides identiques aux séquences de CMVFNY et les tirets (--) représentent les résidus manquants. Les codons ATG et AGT correspondent aux sites d'initiation et terminaison du cadre de lecture. Les différence de nucléotides entre BICMVAR et BICMVGA sont en gras. 


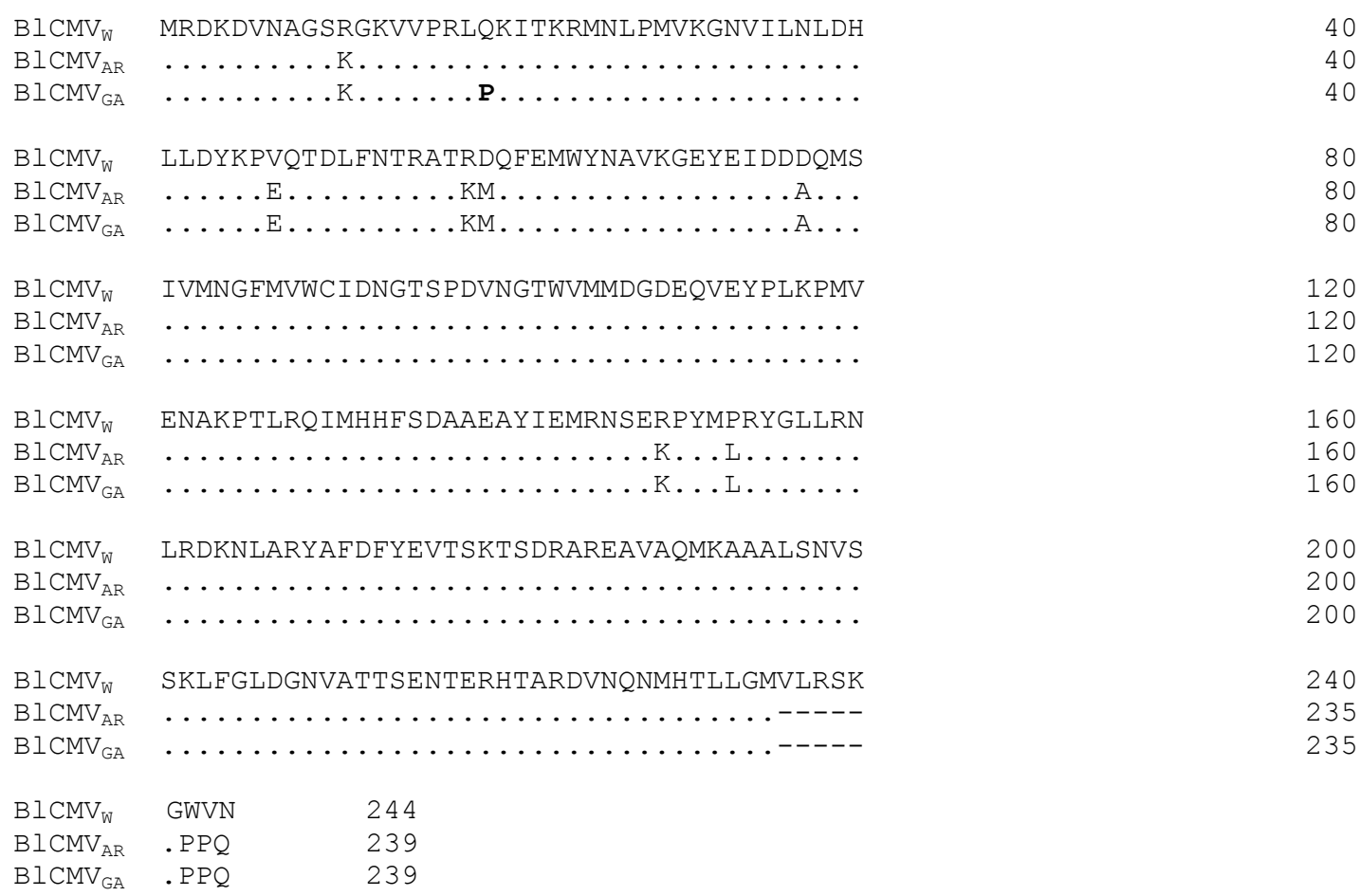

Figure 4 : Comparison of the amino acid sequences of the coat protein genes of the published data for BICMVW (Khan et al., 1993), the Arkansas isolate of BICMV (BICMVAR), and the Georgia isolate of BICMV (BICMVGA).

Dots (...) indicate amino acids identical to the BICMVW sequence, and dashes (---) represent missing residues. Amino acids differences are in bold.

Comparaison des séquences d'acides aminés des gènes des protéines de capside de I'isolat de BICMVW publié (Khan et al., 1993), de l'isolat de BICMV de l'Arkansas (BICMVAR), et de l'isolat de BICMV de la Georgie (BICMVGA).

Les points (...) indiquent les acides aminés identiques à la séquences de BICMVW, et les tirets (---) les résidus manquants. Les différences d'acides aminés entre BICMVAR et BICMVGA sont en gras.

\section{DISCUSSION}

Cowpea stunt disease, first observed in Georgia, was found later in Arkansas (Anderson et al., 1994). It was therefore necessary to compare the cowpea stunt-causing isolates of CMV and $\mathrm{BICMV}$ from both geographical locations. Based on a biological study conducted previously, it was found that although the four types of mixed infections resulted in similar cowpea stunt disease symptoms on inoculated cowpea plants, the different virus accumulation patterns differed (Diallo, 1998). It was concluded from that study that the CMV and BICMV isolates associated with the cowpea stunt disease from Arkansas and Georgia, behave differently. In order to not only better understand that difference, but also accurately classify these virus isolates, it was important to characterize them molecularly since in all the previous reports on cowpea stunt viruses, they were classify only based on symptom observation and serology
(Pio-Ribeiro et al., 1978 ; Pio-Ribeiro and Kuhn, 1980; Anderson et al., 1994).

Indeed there used to be a lot of confusion about the taxonomic classification of BICMV. However, with the methods based on the analysis of the nucleotide and amino acid sequences of the coat protein gene and 3'-UTR, it was shown that accurate results could be obtained (Khan et al., 1990, 1993; Frenkel et al., 1989, 1991; Lana et al., 1988; Van der Vlugt, 1993). These methods were therefore used in this study to characterized and compare the Arkansas and Georgia isolates of BICMV, respectively, $B_{\text {BICMV }}$ AR and BICMV $V_{G A}$. Nucleotide sequence comparison of the coat protein genes and $3^{\prime}$ untranslated regions of BICMV isolates from Arkansas and Georgia revealed only one nucleotide difference which in turn resulted in one amino acid difference in the coat protein. The coat protein of both BICMV isolates displayed more than $93 \%$ homology with $B I C M V w$. This result confirms that the potyvirus 
isolates involved in the cowpea stunt disease in Arkansas and Georgia are indeed BICMV. However, only one nucleotide difference was found between the BICMV isolates, indicating that there was not much variation in the BICMV isolates from both locations and associated with the disease.

Similarly, since it was shown that the analysis of the nucleotide sequence of the coat protein gene and 3' flanking region of CMV provides a good differentiation between isolates (Quemada et al., 1989; Rizos et al., 1992), this method was also used in this study. Nucleotide sequence comparison of the coat protein genes and 3' flanking regions of both CMV isolates showed 8 nucleotides differences between the Arkansas and the Georgia isolates, resulting in $90 \%$ homology with CMVs belonging to subgroup I and only $70-80 \%$ homology with subgroup II CMVs. Therefore, It could be concluded that based on nucleotide sequence comparison, both $\mathrm{CMV}$ isolates belong to subgroup I of CMV. Amino acids comparison revealed only two nucleotides differences between the cowpea stunt disease-causing isolates of CMV from Arkansas and Georgia.

\section{CONCLUSION}

Cucumber mosaic virus (CMV) isolates from Arkansas and Georgia belong to CMV subgroup I and have seven nucleotides differences, resulting in two amino acids differences in their coat protein genes and 3' flanking regions. For the BICMV isolates from both locations, there was only one nucleotide difference, leading to one amino acid difference in the coat protein genes and 3'-untranslated region. This result shows that even with differences in amino acids, the different virus isolates can still cause the disease. The epidemiological implication of the results could be even more important if it is shown that all mixed infections involving any strain or isolate of CMV and BICMV could cause cowpea stunt disease.

This work is the first report of the taxonomic classification of cowpea stunt-causing viruses from Arkansas and Georgia based on nucleotide and amino acid sequences analysis.

\section{REFERENCES}

ANDERSON (E. J.), (A. S.) KLINE, (K. S.) KIM, (S. C.) GOEKE and (C.W.) ALBRITTON. 1994. Identification of cowpea stunt disease in south central Arkansas. Ark. Farm Res. 43 (2) : 14-15.

BOCK (K. R.) and (M.) CONTI. 1974. Cowpea aphidborne mosaic virus. CMI/AAB. Descriptions of plant viruses, No. 134.

BRANTLEY (B. B.). 1976. Coronet, a new southern pea variety. GA. Agric. Exp. Stn. Res. Rep. 220.

DEVERGNE (J. C.) and (L.) CARDING. 1973. Contribution à l'étude du virus de la mosaïque du concombre (CMV). IV. Essai de classification de plusieurs isolats sur la base de leur structure antigénique. Ann. Phytopathol. $5:$ 409-430.

DIALLO (A. H.). 1998. Cowpea stunt : a model system for the study of plant virus synergism. University of Arkansas, Ph.D. Thesis Fayetteville. 180 p.

EDWARDS (M. C.) and (D) GONSALVES. 1983. Grouping of seven biologically defined isolates of cucumber mosaic virus by peptide mapping. Phytopathology 73 : 11171120.

EDWARDSON (J. R.), (F. W.) ZETTLER, (R.- G.) CHRISTIE and (I. R.) EVANS. 1972. A cytological comparison of inclusions as a basis for distinguishing two filamentous legume viruses. J. Gen. Virol. 15 : 113-118.

FRENKEL (M. J.), (C. W.) WARD and (D. D.) SHUKLA. 1989. The use of 3 ' non-coding nucleotide sequences in the taxonomy of potyviruses : Application to watermelon mosaic virus 2 and soybean mosaic virusN. J. Gen. Virol. $70: 2775-2783$.

FRENKEL (M. J.), (J. M.) DILKA, (N. M.) MCKEEN, (P. M.) STOKE, (J. M. JR.) CLARK, (D. D.) SHUKLA and (C. W) WARD. 1991. Unexpected sequence diversity in the amino-terminal end of the coat protein of strains of sugarcane mosaic virus. J. Gen. Virol. $72:$ :237-242.

GAY (J. D.) and (E. E.) WINSTEAD. 1970. Seedborne viruses and fungi from southern pea seed grown in eight States. Plant Dis. Rep. 54 : 243-245. 
GOUDA (T. J.) and (R. H.) SYMONS. 1978. The use of hybridization analysis with complementary DNA to determine the RNA sequence homology between strains of plants viruses: its application to several strains of cucumoviruses. Virology 88 : 361-370.

HARRISON (A. N.) and (R. T.) GUDAUSKAS. 1968 a. Effects of some viruses on growth and seed production of two cowpea cultivars. Plant Dis. Rep. 52 : 509-511.

HARRISON (A. N.) and (R. T.) GUDAUSKAS. 1968 b. Identification of viruses isolated from cowpea in Alabama. Plant Dis. Rep. 52 : 34-36.

KAPPER (J. M.) and (H. E.) WATERWORTH. 1981. Cucumoviruses : 257-332. In : Handbook of plant virus infections and comparative diagnosis (Kurstak E., ed) Elsevier/NorthHolland, New York.

KHAN (J. A.) (D.) LOHUIS, (R.) GOLDBACH and (J.) DIJKSTRA. 1990. Distinction of strains bean common mosaic virus using antibodies to $-\mathrm{N}$ and $-\mathrm{C}$ or $\mathrm{N}$-terminal peptide domains of coat protein. Ann. Appl. Biol. 117 : 583-593.

KHAN (J. A.) (D.) LOHUIS, (R.) GOLDBACH and (J.) DIJKSTRA. 1993. Sequence data to settle the taxonomic position of bean common mosaic virus and blackeye cowpea mosaic virus isolates. J. Gen. Virol. $74: 2243-2249$.

KUHN (C. W.). 1964. Separation of cowpea virus mixtures. Phytopathology 54 : 739-740.

KUHN (C. W.) (B.B.) BRANTLEY and (G.) SORVELL. 1965. Immunity to bean yellow mosaic virus in cowpea. Plant Dis. Rep. 49 : 879881.

LANA (A. F.), (H.) LOHUIS, (L.) BOS and (J.) DIJKSTRA. 1988. Relationship among strains of bean common mosaic virus and blackeye cowpea mosaic members of the potyvirus group. Ann. Appl. Biol. 113 : 493505.

MATTHEWS (R. E. F.). 1991. Plant Virology, Third edition. Academic Press Inc., New York,
$835 \mathrm{p}$.

MOSSOP (P. W.) and (R. I. B.) FRANCKI. 1977. Association of RNAs with aphid transmission of cucumber mosaic virus. Virology 81 : 177-181.

OWEN (J.) and (P.) PALUKAITIS. 1988. Characterization of cucumber mosaic virus. I. Molecular heterogeneity mapping of RNA 3 in eight CMV strains. Virology 166 : 495-502.

PALUKAITIS (P.), (M. J.) ROOSSINCK, (R. G.) DIETZEN and (R. I. B.) FRANCKI. 1992. Cucumber mosaic virus. Adv. Vir. Res. 41 : 281-348.

PAPPU (S. S.), (R.) BRAND, (H. R.) PAPPU, (E. P.) RYBICKI, (K. H.) GOUGH, (M. J.) FRENCKEL and (C. L.) NIBLETT. 1993. A polymerase chain reaction method adapted for selective amplification and cloning of $3^{\prime}$ sequences of potyviral genomes : application to dasheen mosaic virus. J. Virol. Meth. $4: 9-20$.

PIAZZOLLA (P.), (J. R.) DIAZ-RUIZ and (J. M.) KAPPER. 1979. Nucleic acid homologies of eighteen cucumber mosaic virus isolates determined by competition hybridization. J. Gen. Virol. 45 : 361-369.

PIO-RIBEIRO (G.), (S. D.) WYATT and (C .W) KUHN. 1978. Cowpea stunt : a disease caused by a synergistic interaction of two viruses. Phytopathology $68: 1260-1265$.

PIO-RIBEIRO (G.) and (C. W.) KUHN. 1980. Cowpea stunt : heterogeneous and differential reactions of cowpea cultivars. Phyto-pathology 70 : 244-249.

QUEMADA (H.), (C.) KEARNEY, (D.) GONSALVES and (J. L.) SLIGHTON. 1989. Nucleotide sequences of the coat protein genes and flanking regions of cucumber mosaic virus isolates using the polymerase chain reaction. J. Gen. Virol. $73: 2099-2103$.

RIZOS (H.), (L. V.) GUNN, (R. D.) PARE and (M. R.) GILLINGS. 1992. Differentiation of cucumber mosaic virus isolates using the polymerase chain reaction. J. Gen. Virol. 73 : 2099-2103. 
SANGER (F.), (S.) MILKEN and (A. R.) COULSON. 1977. DANN sequencing with chain terminating inhibitors. Proc. Nat. Acad. Sci. USA. $74: 5463-5467$

SHUKLA (D. D.) and (C. W.) WARD. 1988. Amino acid sequence homology of coat protein as a basis for identification and classification of the potyvirus group. J. Gen. Virol. 69 : 2703-2710.

SHUKLA (D. D.) and (C. W.) WARD. 1989. Identification and classification of potyviruses on the basis of coat protein sequence data and serology. Arch. Virol. 106 : 171-200.

SINGH (B. B.), (O. L.) CHAMBLISS and (B.) SHARMA. 1997. Recent advances in cowpea breeding. B.B. Singh, D.R. Mohan Raj, K.E. Dashiel and L.E.N. Jackai, eds. IITA, Ibadan, Nigeria.
TAIWO (M. A.), (D.) GONSALVES, (R.) PROVIDENTI and (H. D) THURSTON. 1982. Partial characterization and grouping of isolates of blackeye mosaic cowpea aphid-borne mosaic viruses. Phytopathology 72 : 590596.

VAN DER VLUGT (R. A. A.), (J.) LEUNISSEN and (R.) GOLDBACH. 1993. Taxonomic relationship between distinct potato virus $Y$ isolates based on detailed comparisons of the viral coat proteins and 3'-nontranslated regions. Arch. Virol. $131: 361-375$.

ZETTLER (F. W.) and (I. R.) EVANS. 1972. Blackeye cowpea mosaic virus in Florida : Host range incidence in certified cowpea seed. Proc. Fla. State Hortic. Soc. 85 : 99-101. 